\title{
Inhibition of PCSK9 protects against radiation- induced damage of prostate cancer cells
}

\author{
This article was published in the following Dove Press journal: \\ OncoTargets and Therapy \\ 12 April 2017 \\ Number of times this article has been viewed
}

\section{Si-Shun Gan* \\ Jian-Qing Ye* \\ Lei Wang \\ Fa-Jun Qu \\ Chuan-Min Chu \\ Yi-Jun Tian \\ Wei Yang \\ Xin-Gang Cui}

Department of Urinary Surgery of Third Affiliated Hospital, Second Military Medical University, Shanghai, People's Republic of China

*These authors contributed equally to this work
Correspondence: Xin-Gang Cui Department of Urinary Surgery of Third Affiliated Hospital, Second Military Medical University, No 700, North Moyu Road, Jiading District, Shanghai 201805,

People's Republic of China

Email cuixg2016@sohu.com
Background: Proprotein convertase subtilisin/kexin type 9 (PCSK9) is a protein expressed primarily in the liver, formerly known to maintain plasma lipid homeostasis by regulating lowdensity lipoprotein receptor levels, and its exact role in the radioresistance of prostate cancer (PCa) remains unclear. We aim to investigate the function of PCSK9 in the radioresistance of PCa cells.

Methods: PCSK9 small interfering RNA (siRNA) was introduced into the PCa cells by transient transfection. Then, cells were exposed to ionizing radiation (IR) at indicated dose rates. Cell damage was detected using cell counting kit-8 (CCK-8) and Hoechest 33342/propidium iodide (PI) staining. Rhodamine-123 (Rho-123) dye was used to assay mitochondrial membrane potential alteration. Western blot was used to detect the apoptosis-related protein expression. Results: PCSK9 siRNA treatment significantly protected PCa cells from IR-induced cell damage, including enhancing cell viability, reducing apoptosis, and inhibiting MMPs. Moreover, PCSK9 siRNA repressed the increase of cytochrome C (cyto C), caspase-3, and B-cell leukemia/ lymphoma 2 (Bcl-2)-associated X (Bax) expressions induced by IR and promoted Bcl-2 expression, which might partially interpret the radioprotective role of PCSK9 siRNA in PCa cells.

Conclusion: PCSK9 might impact on radiosensitivity through mitochondrial pathways and serve as a novel therapeutic target for PCa patients.

Keywords: PCSK9, prostate cancer, radioresistance, mitochondrial pathway

\section{Background}

Prostate cancer $(\mathrm{PCa})$ is among the most common cancers in men and a major cause of cancer-related deaths worldwide, with one in 16 afflicted men dying from this disease every year in Western countries. ${ }^{1,2}$ Currently, treatment options, including surgery, radiotherapy, and hormonal therapy, are usual methods for treating this disease. The first-line modality for PCa patients with localized disease is radiotherapy $-\sim 25 \%$ of PCa patients have some form of radiotherapy incorporated into their treatment regimen. ${ }^{3}$ Technical advances in radiotherapy delivery, including image-guided intensitymodulated radiotherapy (IG-IMRT), have achieved the purpose of higher radiation dose delivered to the prostate, which improves biochemical control. ${ }^{4,5}$ However, the risk of recurrence following radiotherapy remains high for quite a number of $\mathrm{PCa}$ patients, and tumor radioresistance remains a major clinical problem. ${ }^{6,7}$ Therefore, it is necessary to identify the key factors involved in regulating the sensitivity of PCa cells to radiotherapy, which finally contributes to improve the clinical outcomes and prognosis of this disease.

Proprotein convertase subtilisin/kexin type 9 (PCSK9) is a protein expressed primarily in the liver and plays an important role in cholesterol metabolism by regulating low-density lipoprotein (LDL) receptor levels. It was first described in 2003, when 
it was termed as neural apoptosis-regulated convertase 1 (NARC-1) due to its role in liver regeneration and neuronal differentiation. ${ }^{8}$ In most conditions, PCSK9 is thought to mainly maintain plasma lipid homeostasis, and its inhibitory antibodies alirocumab, evolocumab, and bococizumab have already been in commercial development to reduce LDL cholesterol. ${ }^{9,10}$ They are a class of novel lipid-lowering drugs in the clinic, with similar pharmacological actions as statins. For statins, there are numerous reports regarding their roles in $\mathrm{PCa}$ cells and radiotherapy of PCa patients. ${ }^{11,12} \mathrm{With}$ the increasing investigations regarding the other roles of PCSK9, we realized its novel functions, including regulating cell cycle, inflammatory response, and tumor metastasis. ${ }^{13-16}$ In vivo studies also support the roles of PCSK9 in these processes. However, there are still no studies concerning the implication of PCSK9 in the radiotherapy of cancer cells, especially in PCa cells. Whether its inhibitors would exert a protective effect or the opposite? Given that PCSK9 inhibitors might be widely used in the clinic, it is of practical significance and clinical value to investigate the relationship between PCSK9 and PCa.

In this study, we took advantage of PCSK9 small interfering RNA (siRNA) to learn about its role in radiosensitivity of $\mathrm{PCa}$ cells. We found that PCSK9 may negatively regulate IR-induced apoptosis through mitochondrial pathway in PCa cells.

\section{Methods}

\section{Cell culture}

Two PCa cell lines LnCap and PC-3 were ordered from the Cell Bank of Shanghai Institutes for Biological Sciences, Chinese Academy of Sciences (Shanghai, China). Cells were routinely maintained in Roswell Park Memorial Institute1640 medium (HyClone, Beijing, China) supplemented with $10 \%$ fetal bovine serum (FBS) in a humidified atmosphere with $5 \% \mathrm{CO}_{2}$ at $37^{\circ} \mathrm{C}$.

\section{Irradiation exposure}

Ionizing radiation (irradiation, IR) exposure was conducted using an irradiator with a cobalt Co60 source (Co-V, Theratron 780; MDS Nordion, Ottawa, ON, Canada) at indicated dose rates: 0-12 Gy/min. Afterward, cells were returned for incubation in normal culture medium.

\section{siRNA design and transfection}

PCSK9 siRNAs and control siRNA (a universal negative control) were chemically synthesized and purified by GenePharma (Shanghai, China). The sequences were as follows: PCSK9 siRNA-1: 5'-CCUGGAGUUUAUUCGGAAAdTdT-3',
PCSK9 siRNA-2: 5'-GGCAGAGACUGAUCCACU UdTdT-3'. To detect the expression of PCSK9, PCa cells were grown in six-well plates until 30\%-50\% confluency and transfected with PCSK siRNAs (50 nmol/L) by Lipofectamine 2000 (Invitrogen, Carlsbad, CA, USA) according to the manufacturer's protocol. Cells transfected with nonsense negative control siRNA were used as blank.

\section{Real-time PCR}

Total RNA was isolated from cells by TRIzol (Invitrogen). After measuring the RNA concentration, reverse transcription was performed to generate cDNA using random primers and Transcriptor First Strand cDNA Synthesis Kit (Takara, Dalian, China). Real-time PCR was done on a LightCycler 480 (Roche, Basel, Switzerland) by applying the SYBR Green PCR Kit (Toyobo, Osaka, Japan). GAPDH was used as the control. Gene expression was calculated by the $2^{-\Delta \Delta \mathrm{Ct}}$ method. Primer sequences for PCSK9 and GAPDH were as follows: PCSK9 forward, GGAACCTGGAGCGGATTACC, PCSK9 reverse, TTTCCCGGTGGTCACTCTGT; GAPDH forward, GGAGTCCACTGGCGTCTTCA, GAPDH reverse, TTCACACCCATGACGAACATG.

\section{Western blot}

Cells were lysed with radio-immunoprecipitation assay buffer supplemented with protease inhibitors. Protein amounts were determined by bicinchoninic acid assay kit (Solarbio, Beijing, China), and equal quantities of protein were loaded onto $10 \%$ Sodium dodecyl sulfate-polyacrylamide gel electrophoresis followed by transferring to polyvinylidene fluoride membranes. After blocking, the membranes were incubated with indicated antibodies. The antibody against $\beta$-actin (Santa Cruz Biotechnology, Dallas, TX, USA) was used as the loading control. Specific protein expression was detected by using the chemiluminescence reagents (Pierce, Rockford, IL, USA). The quantification of specific band density was done by Quantity One software (Bio-Rad, Hercules, CA, USA) with normalization to the $\beta$-actin signal.

\section{Cell viability detection}

Cell viability was detected by the cell counting kit-8 (CCK-8) reagent (Dojindo, Kumamoto, Japan). Briefly, cells were digested by trypsin and seeded onto a 96-well plate post siRNA transfections and IR exposure, at a density of $5 \times 10^{3}$ cells/well. After incubation with normal culture for $24 \mathrm{~h}, 10 \mu \mathrm{L}$ of CCK-8 reagent was added into wells and kept for $2 \mathrm{~h}$ at $37^{\circ} \mathrm{C}$. The absorbance was measured at $450 \mathrm{~nm}$ in a microplate reader. 


\section{Cell morphological analysis by Hoechest 33342/PI staining}

siRNA-transfected cells were seeded in 24-well plates at the same density overnight and treated with 8 Gy IR. Then, cells were fixed and double stained with Hoechst 33342 and propidium iodide (PI). Apoptotic cells indicated by strong blue signal and dead cells indicated by PI-positive (red) signal were visualized. Images were taken using a fluorescence microscope (Leica, Wetzlar, Germany).

\section{Evaluation of mitochondrial membrane potential (MMP)}

Rhodamine-123 (Rho-123) dye (Dojindo) was used to assay MMP alteration. Following IR treatment, cells were stained with $1 \mu \mathrm{M}$ working solution containing Rho-123 and incubated at $37^{\circ} \mathrm{C}$ in the incubator for $30 \mathrm{~min}$, followed by washing with PBS and visualizing under a $535 \mathrm{~nm}$ fluorescence microscope or analyzed by flow cytometry.

\section{Statistics}

Data were expressed as mean \pm SD and analyzed using Student's $t$-test or one-way ANOVA to evaluate the statistical significance. $P$-values $<0.05$ were considered statistically significant. All experiments were repeated independently at least three times.

\section{Results}

\section{Cell viability depressed by IR exposure is} attenuated by PCSK9 siRNA in PCa cells

The existence of radioresistance frequently impairs the therapeutic effects of human malignancies. To test whether PCSK9 is associated with radioresistance in PCa cells, we first transfected two PCa cell lines LnCap and PC-3 cells with two independent siRNAs targeting PCSK9 and then exposed them to increasing doses of IR. As shown in Figure 1A and B, the treatment of two PCSK9 siRNAs significantly reduced the endogenous expression level of PCSK9 mRNA and protein, in which the siRNA-1 had the better knockdown efficiency. Therefore, we utilized this siRNA as the PCSK9 inhibitor. Following IR exposure ( $0,4,6,8$, and 12 Gy), CCK-8 assay showed that cell viability was reduced in a dose-dependent manner, and high doses of IR ( 8 and 12 Gy) markedly decreased cell viability in both LnCap and PC-3 cells
A

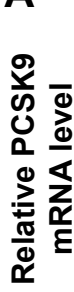

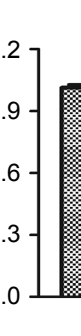

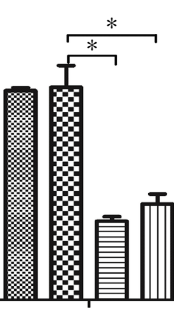

LnCap

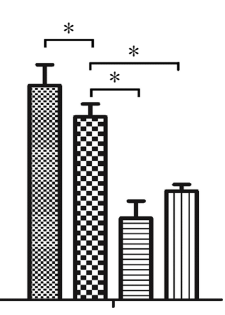

PC-3

\section{B}

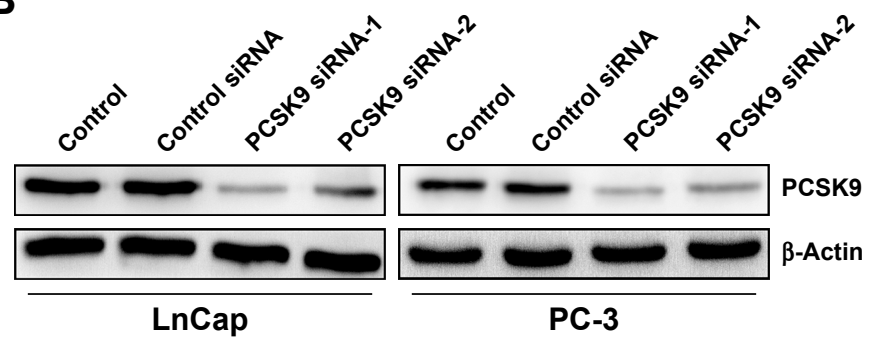

Control Control siRNA

ㅁ PCSK9 siRNA-1 س

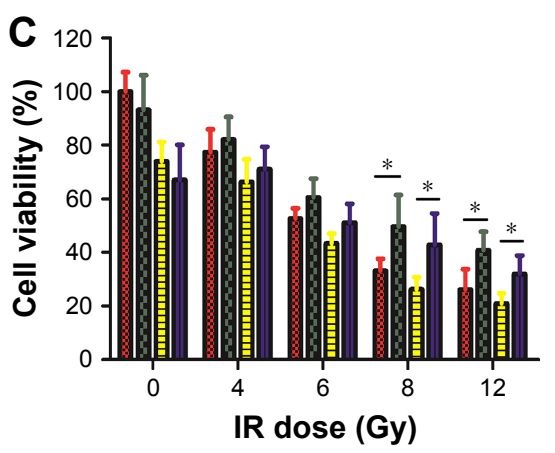

LnCap + control siRNA

G LnCap + PCSK9 siRNA

曰 PC-3 + control siRNA

PC-3 + PCSK9 siRNA

Figure I PCSK9 siRNA treatment promotes cellular viability in cells exposed to high doses of IR.

Notes: (A) LnCap cells (left) and PC-3 cells (right) were, respectively, transfected with PCSK9 siRNAs (PCSK9 siRNA-I and siRNA-2) and control siRNA. After 48 h, the efficiency of PCSK9 knockdown was determined by real-time PCR. (B) PCSK9 protein expression in the siRNA-treated cells was analyzed by Western blot. $\beta$-Actin was included as a loading control. (C) After $24 \mathrm{~h}$ of PCSK9 siRNA pretreatment, relative cellular viability of the indicated cell lines after treatment with increasing doses of IR was determined by CCK-8 assay. Data are expressed as the mean \pm SD $(* P<0.05)$.

Abbreviations: CCK-8, cell counting kit-8; IR, ionizing radiation; PCSK9, proprotein convertase subtilisin/kexin type 9; siRNAs, small interfering RNAs; SD, standard deviation. 
(Figure 1C). However, the depression in cell viability by IR exposure was reversed by PCSK9 siRNA treatment (Figure 1C). In other words, PSCK9 inhibitor significantly promoted PCa cell survival, or PCSK9 inhibitor induced radioresistance post-IR exposure.

\section{Apoptosis induced by IR is depressed in PCa cells treated by PCSK9 siRNA}

We then investigated whether PCSK9 siRNA impacted on IR-induced cell apoptosis in PCa cells, since apoptosis is the main consequence of cell damage induced by IR exposure or radiotherapy. We found that IR ( 8 Gy) exposure induced significant apoptosis in LnCap and PC-3 cells, which was reflected by the results of Hoechest 33342/PI staining. Blue emission by Hoechest 33342 was much brighter, and PI staining was positive in the IR-exposed cells than in the control siRNA-treated cells, indicating the presence of apoptotic and dead cells, respectively (Figure 2A and B). However, in PCSK9 siRNA-pretreated cells, IR-induced apoptosis was attenuated significantly and this effect was more obvious in LnCap cells (Figure 2A and $\mathrm{B})$. These data indicated that the inhibition of PCSK9 increased the radioresistance of $\mathrm{PCa}$ cells through the antiapoptotic effects.

\section{Inhibition of IR-induced apoptosis in PCSK9 siRNA-treated cells involves mitochondrial pathway}

As is known, apoptosis generally includes intrinsic (mitochondrial) or extrinsic pathway or both. To test for the involvement of mitochondrial pathway, the effects of PCSK9 siRNA on IR-induced alterations of MMP in LnCap cells were assessed. As shown in Figure 3A, fluorescence intensity of Rho-123 indicative of MMPs exhibited a moderate depolarization of MMP by 8 Gy IR exposure. PCSK 9 siRNA pretreatment decreased MMPs as visualized by less Rho-123 signal (Figure 3A). In addition, flow cytometry was conducted to quantify the fluorescence intensity of Rho-123. As shown in Figure 3B and C, the pretreatment of LnCap cells with PCSK9 siRNA for $24 \mathrm{~h}$ resulted in a decreased fluorescence intensity of Rho-123 postradiotherapy, as shown in Figure 3A. These data supported that the inhibition of IR-induced apoptosis by PCSK9 siRNA involved mitochondrial pathway in $\mathrm{PCa}$ cells.

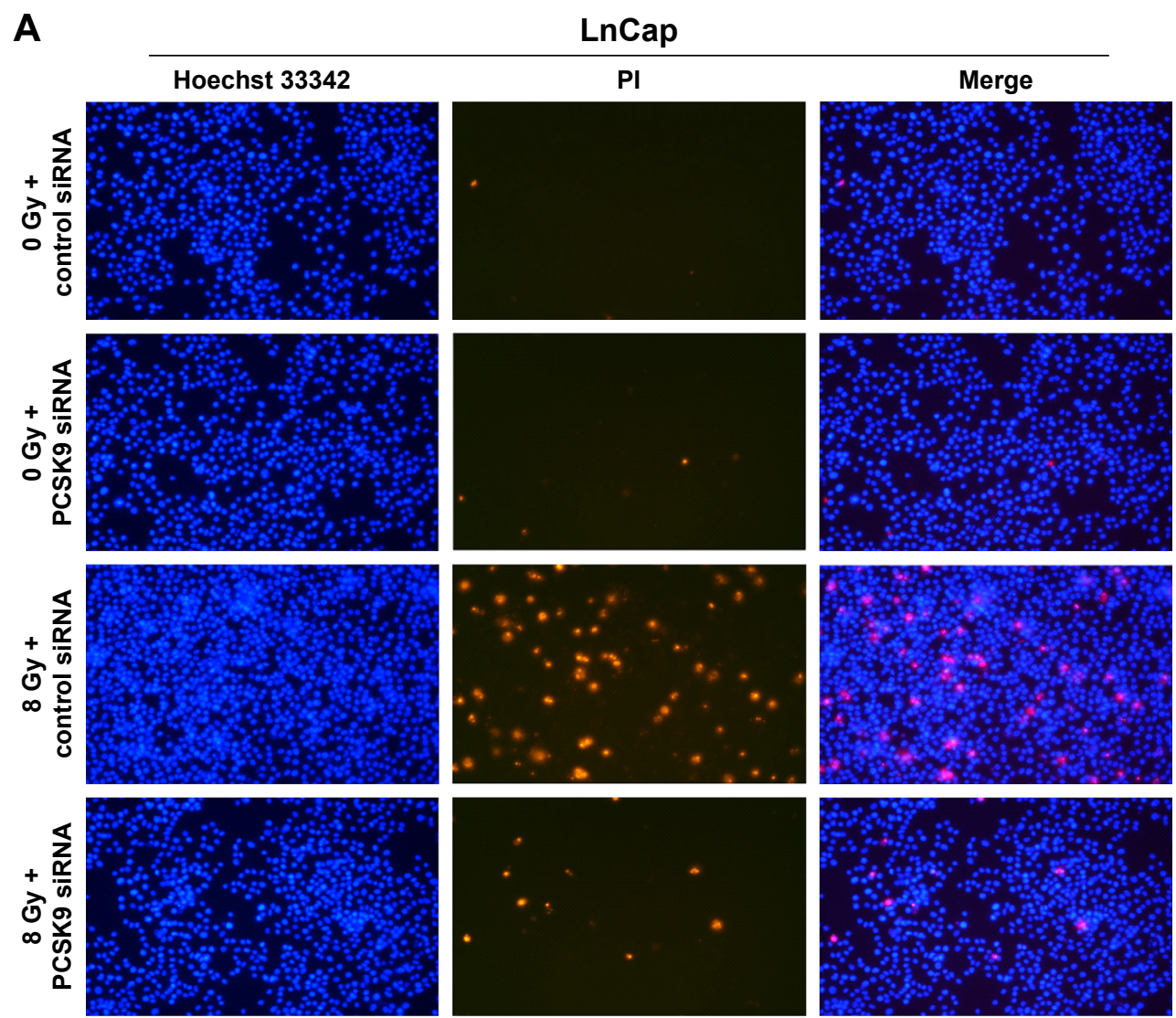

Figure 2 (Continued) 


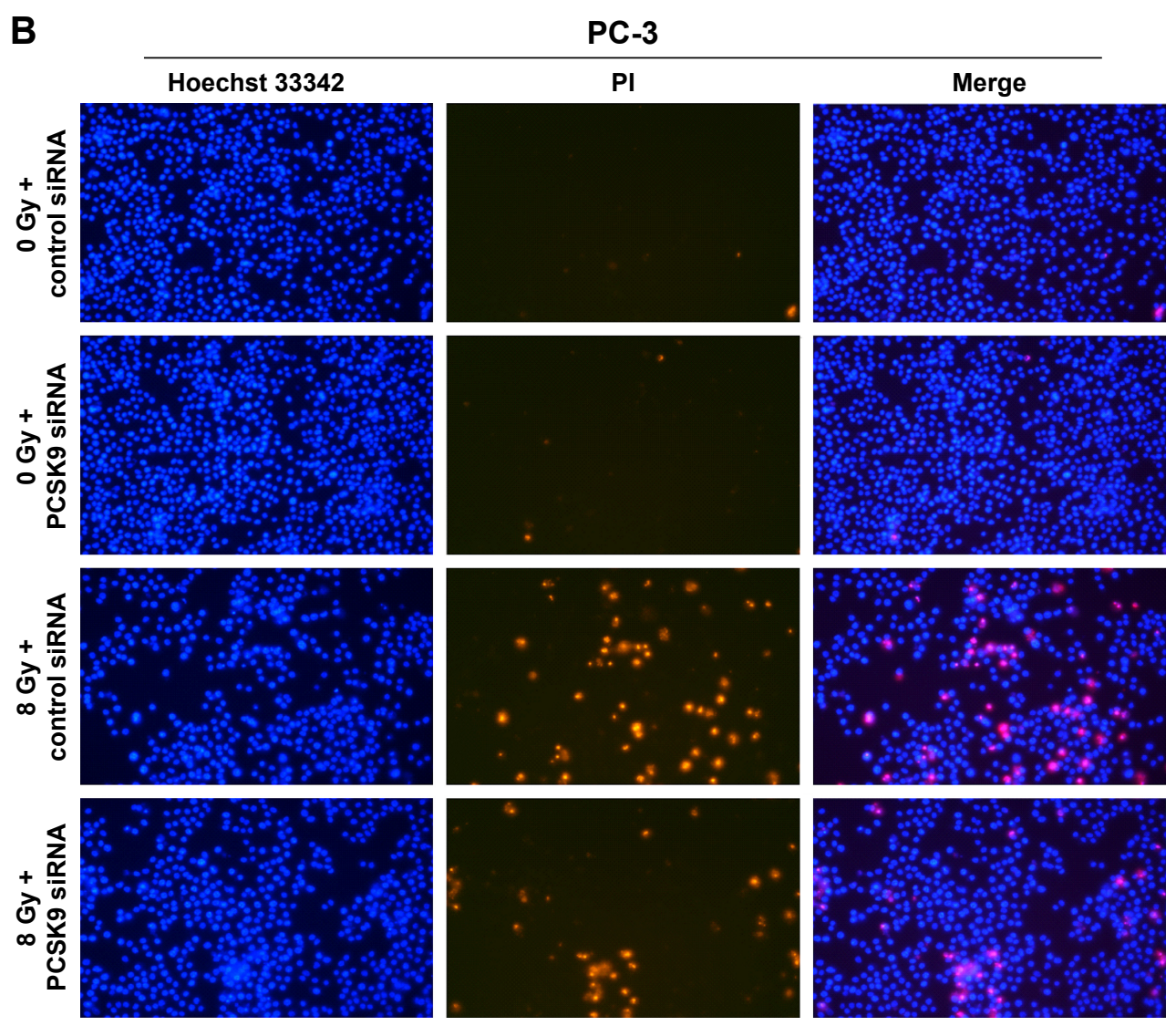

Figure 2 Inhibition of PCSK9 increases radioresistance in PCa cells exposed to IR.

Notes: After PCSK9 was inhibited by $24 \mathrm{~h}$ of siRNA pretreatment, LnCap cells (A) and PC-3 cells (B) were exposed to 0 or 8 Gy of IR. Cellular apoptosis was revealed by Hoechest 33342/PI staining. Apoptotic cells exhibited blue-positive staining, whereas dead cells exhibited red-positive staining. PCSK9 siRNA treatment alone did not cause cell apoptosis. IR-induced apoptosis was attenuated by PCSK9 siRNA treatment. Magnification $\times 200$.

Abbreviations: IR, ionizing radiation; PCSK9, proprotein convertase subtilisin/kexin type 9; PI, propidium iodide; siRNA, small interfering RNA.

\section{Cyto C, caspase-3, B-cell leukemia/ lymphoma 2 (Bcl-2)-associated X (Bax), and $\mathrm{Bcl}-2$ proteins are involved in PCSK9 siRNA-induced radioresistance}

To clarify the molecular mechanism, which mediates the inhibition of PCSK9 siRNA-mediated PCa cell apoptosis by radiotherapy, the expression levels of mitochondrial apoptosis-associated proteins, such as cytochrome C (cyto C), caspase-3, Bax, and Bcl-2, were determined by Western blot. We also analyzed the expression of PCSK9 protein in the post-IR time period. Our data revealed that in the normal LnCap cells post-IR, PCSK9 expression remained steady (Figure 4A and B), whereas we observed increased cyto $\mathrm{C}$, caspase-3, and Bax expressions in a time-dependent manner, with peaks at $8 \mathrm{~h}$ post-IR (Figure 4A, C, and D). We also found that Bcl-2 was decreased in this period, with the trough at $12 \mathrm{~h}$ post-IR (Figure $4 \mathrm{~A}$ and $\mathrm{E}$ ). In contrast, in the IR-exposed cells, the levels of cyto C, caspase-3, and Bax were significantly decreased, whereas Bcl-2 was significantly increased in PCSK9 siRNA-treated cells compared to that in the control siRNA-treated cells (Figure 4F and G). These results indicated that the radioresistance triggered by PCSK 9 siRNA treatment is mediated by cyto $\mathrm{C}$, caspase-3, Bax, and Bcl-2 signaling in PCa cells.

\section{Discussion}

PCSK9 is a proprotein convertase, which is involved in the degradation of LDL receptors in the liver. It is also named NARC-1, raising the possibility that PCSK9 might be involved in apoptosis. ${ }^{17} \mathrm{In}$ fact, animal studies have suggested roles for PCSK9 in nonhepatic tissues. These include inflammation, intestinal and adipocyte lipid metabolism, cell apoptosis, and regulation of blood pressure. ${ }^{18}$

Recently, the implication of PCSK9 in cell apoptosis has been frequently evoked. For instance, the lack of PCSK9 had been found to promote apoptosis during liver regeneration. ${ }^{19}$ A microarray study showed that PCSK $9^{\mathrm{D} 347 \mathrm{Y}}$ overexpression resulted in downregulation of proapoptotic genes in 
A

Control siRNA
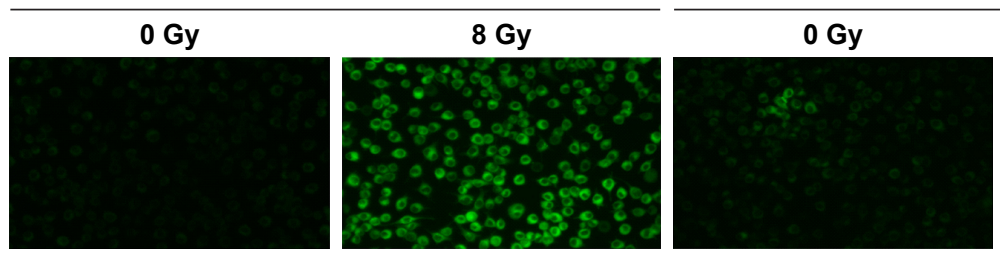

PCSK9 SIRNA

B

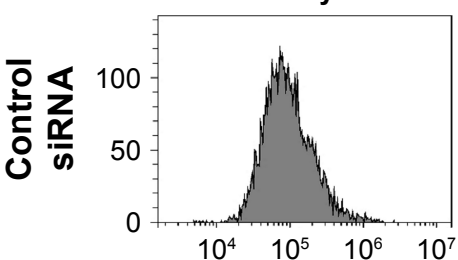

$8 \mathrm{~Gy}$
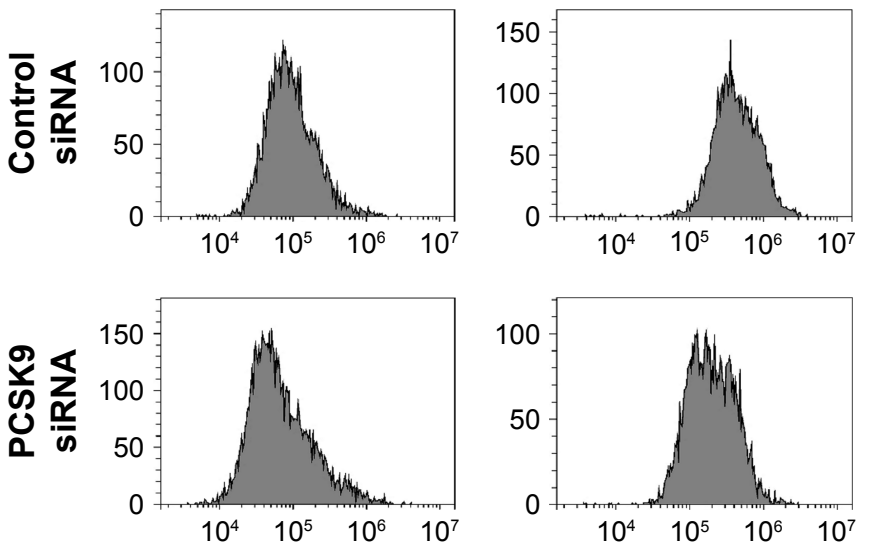

C

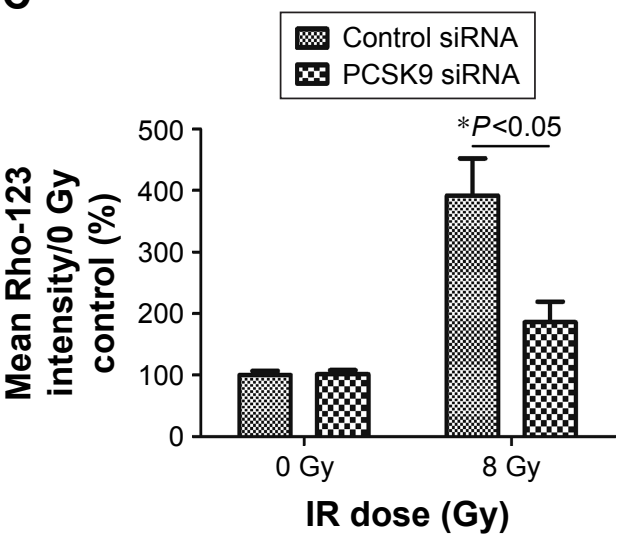

Figure 3 Inhibition of PCSK9 decreases MMP in LnCap cells exposed to IR.

Notes: LnCap cells transfected with control or PCSK9 siRNA were treated with 0 or 8 Gy of IR, respectively. Magnification $\times 400$. (A) MMPs were visualized by Rho- 23 staining, and representative images were given. (B) Flow cytometric analysis of MMP alteration in indicated cells and representative results are shown. (C) Mean Rho- 123 signal assessed by flow cytometry in response to IR exposure. Mean \pm SD of three independent experiments were performed ( $* P<0.05$ vs control siRNA).

Abbreviations: IR, ionizing radiation; MMP, mitochondrial membrane potential; PCSK9, proprotein convertase subtilisin/kexin type 9; Rho-I23, Rhodamine-I23; SD, standard deviation; siRNA, small interfering RNA.

HepG2 cells. ${ }^{20}$ PCSK9 was also reported to inhibit apoptosis through mitochondrial pathway in human neuroglioma U251 cells. ${ }^{21}$ These clues of evidence indicated that PCSK9 might inhibit cell apoptosis. Nevertheless, the implication of PCSK9 in the radiotherapy of cancer cells remains largely unknown. We proved in this study that PCSK9 siRNA exerted a radioresistant or radioprotective function, that is, PCSK9 itself promoted IR-induced cell apoptosis in $\mathrm{PCa}$ cells. We noticed that at normal condition (0 Gy), PCSK9 siRNA did not impact on PCa cell viability or apoptosis. Until exposed by high dose of IR ( 8 Gy), PCSK9 siRNA pretreatment displayed radioresistance, indicated by increased cell viability and inhibition of apoptosis after IR exposure. Our study constitutes the first evidence that PCSK9 has a proapoptotic effect in the radiotherapeutic PCa cells.

Cell apoptosis is an intrinsic physiological process, which leads to self-destruction. Dysregulation of apoptosis is implicated in multiple diseases, including cancers. ${ }^{22}$ Mitochondria has been identified as the major organelle, and its functions include respiration, oxygen metabolism, and enzyme activity regulation and are associated with membrane permeability and MMP. ${ }^{23}$ As is known, mitochondrial apoptosis always involves the release of cyto $\mathrm{C}$ and the activation of caspase- 3 and Bax proteins. ${ }^{24}$ The Bcl-2 family proteins primarily regulate mitochondrial membrane permeabilization, and $\mathrm{Bax} / \mathrm{Bcl}-2$ ratio is always regarded as a criterion for apoptosis. ${ }^{25,26}$ The results from our study revealed that cyto C, caspase-3, and Bax were significantly increased and the ratio of $\mathrm{Bax} / \mathrm{Bcl}-2$ was significantly increased in IR-exposed PCa cells. However, all these alterations were reversed in PCSK9 siRNA-treated PCa cells. This result indicated that PCSK9 might impact on mitochondrial membrane stability and PCSK9 siRNA could exert its radioresistant activity through mitochondrial signaling pathway in PCa cells.

\section{Conclusion}

We found that inhibition of intrinsic PCSK9 could attenuate IR-induced cell damage, including increasing cell viability and inhibiting apoptosis, which involves mitochondrial pathway. Mechanistically, we proved that PCSK9 siRNA repressed the increase of cyto $\mathrm{C}$, caspase-3, and Bax expressions induced by IR and promoted Bcl-2 expression, which might be the possible mechanism underlying its radioprotective role in 
A

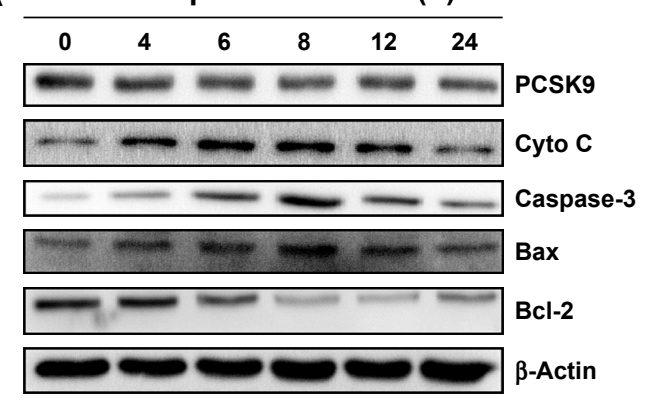

C

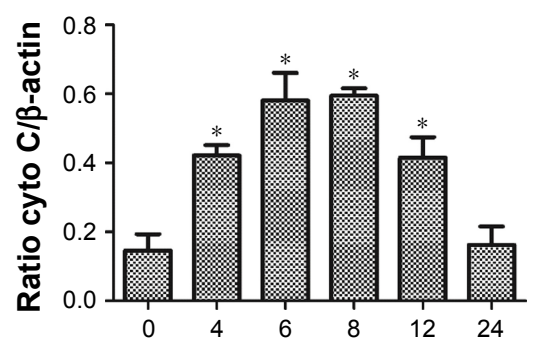

Time of postirradiation (h)
D

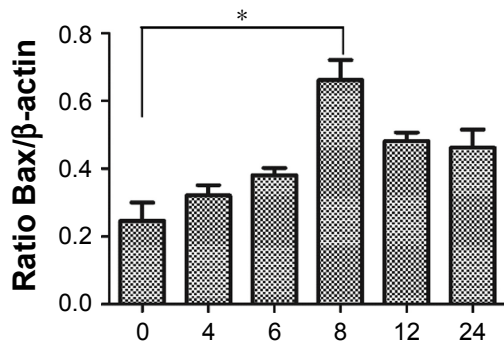

Time of postirradiation (h)

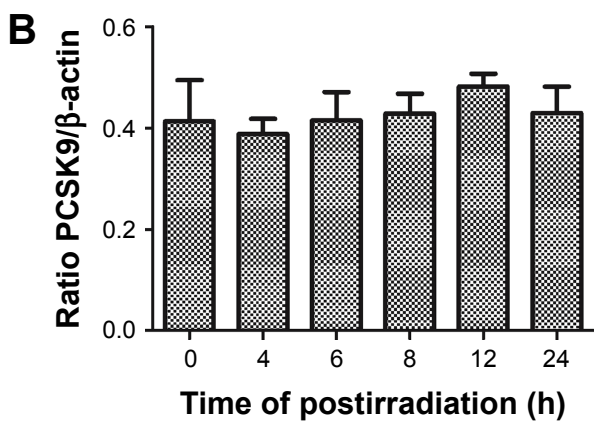

E

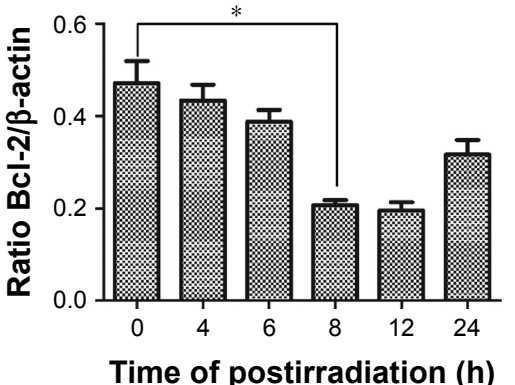

$\mathbf{F}$
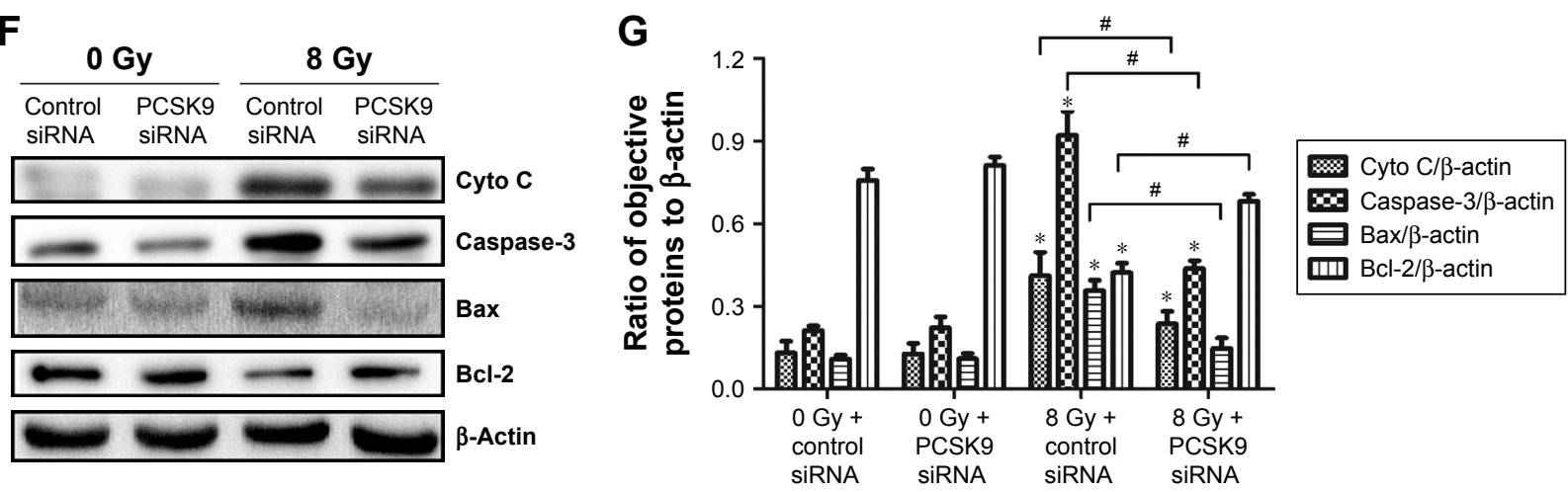

Figure 4 Radioresistance mechanisms of PCSK9 siRNA in LnCap cells.

Notes: (A) LnCap cells were treated with 8 Gy of IR. At different time points post-IR, the expression of PCSK9, cyto C, caspase-3, Bax, and Bcl-2 was measured by Western blot, with $\beta$-actin as the loading control. (B-E) PCSK9, cyto C, Bax, and Bcl-2 protein levels were quantified from three independent experiments as in (A) by measuring the intensity of the indicated protein band relative to the $\beta$-actin (loading) control $\left({ }^{*} P<0.05\right)$. The bars indicate mean $\pm S D(n=3)$. ( $\left.F\right)$ LnCap cells were pretreated with control or PCSK9 siRNA prior to IR ( 0 or $8 \mathrm{~Gy}$ ), and proteins were lysed $24 \mathrm{~h}$ after IR. The expression of cyto C, caspase-3, Bax, and Bcl-2 was measured by Western blot, with $\beta$-actin as the loading control. (G) Cyto C, caspase-3, Bax, and Bcl-2 protein levels were quantified from three independent experiments as in (F). Mean \pm SD of three independent experiments were performed ( ${ }^{*} P<0.05$ vs 0 Gy groups, ${ }^{\sharp} P<0.05$ vs control siRNA groups).

Abbreviations: PCSK9, proprotein convertase subtilisin/kexin type 9; siRNA, small interfering RNA; IR, ionizing radiation; cyto C, cytochrome C; Bcl-2, B-cell leukemia/ lymphoma 2; Bax, Bcl-2-associated X protein; SD, standard deviation.

PCa cells. Taken together, we provided evidence that PCSK9 might represent a promising therapeutic strategy for enhancing the radiosensitivity of $\mathrm{PCa}$ patients.

\section{Acknowledgment}

This study did not receive any specific grant from funding agencies in the public, commercial, or not-for-profit sectors.

\section{Disclosure}

The authors report no conflicts of interest in this work.

\section{References}

1. Cancer Research [webpage on the Internet]. Prostate Cancer Statistics, Cancer Stat. 2011. Available from: http://www.cancerresearchuk.org/ health-professional/cancer-statistics/statistics-by-cancer-type/prostatecancer\#heading-Zero. Accessed March 9, 2017.

2. Ferlay J, Parkin DM, Steliarova-Foucher E. Estimates of cancer incidence and mortality in Europe in 2008. Eur J Cancer. 2010;46(4):765-781.

3. Sriprasad S, Feneley MR, Thompson PM. History of prostate cancer treatment. Surg Oncol. 2009;18(3):1851-1891.

4. Bauman G, Rumble RB, Chen J, Loblaw A, Warde P; Members of the IMRT Indications Expert Panel. Intensity-modulated radiotherapy in the treatment of prostate cancer. Clin Oncol (R Coll Radiol). 2012;24(7): $461-473$. 
5. Lagendijk JJ, Raaymakers BW, van Vulpen M. The magnetic resonance imaging-linac system. Semin Radiat Oncol. 2014;24(3):207-209.

6. Keall PJ, Barton M, Crozier S; Australian MRI-Linac Program, including contributors from Ingham Institute, Illawarra Cancer Care Centre, Liverpool Hospital, Stanford University, Universities of Newcastle, Queensland, Sydney, Western Sydney, and Wollongong. The Australian magnetic resonance imaging-linac program. Semin Radiat Oncol. 2014; 24(3):203-206.

7. Shao YH, Demissie K, Shih W, et al. Contemporary risk profile of prostate cancer in the United States. J Natl Cancer Inst. 2009;101(18): $1280-1283$.

8. Seidah NG, Benjannet S, Wickham L, et al. The secretory proprotein convertase neural apoptosis-regulated convertase 1 (NARC-1): liver regeneration and neuronal differentiation. Proc Natl Acad Sci U S A. 2003;100(3):928-933.

9. Schmidli R. PCSK9 inhibitors - clinical applications. Aust Prescr. 2016; 39(5):168-170.

10. Page MM, Watts GF. PCSK9 inhibitors - mechanisms of action. Aust Prescr. 2016;39(5):164-167.

11. He Z, Mangala LS, Theriot CA, Rohde LH, Wu H, Zhang Y. Cell killing and radiosensitizing effects of atorvastatin in $\mathrm{PC} 3$ prostate cancer cells. $J$ Radiat Res. 2012;53(2):225-233.

12. Oh DS, Koontz B, Freedland SJ, et al. Statin use is associated with decreased prostate cancer recurrence in men treated with brachytherapy. World J Urol. 2015;33(1):93-97.

13. Sun X, Essalmani R, Day R, Khatib AM, Seidah NG, Prat A. Proprotein convertase subtilisin/kexin type 9 deficiency reduces melanoma metastasis in liver. Neoplasia. 2012;14(12):1122-1131.

14. Tang Z, Jiang L, Peng J, et al. PCSK9 siRNA suppresses the inflammatory response induced by oxLDL through inhibition of NF- $\kappa B$ activation in THP-1-derived macrophages. Int J Mol Med. 2012;30(4):931-938.

15. Lan H, Pang L, Smith MM, et al. Proprotein convertase subtilisin/kexin type 9 (PCSK9) affects gene expression pathways beyond cholesterol metabolism in liver cells. J Cell Physiol. 2010;224(1):273-281.
16. Mbikay M, Sirois F, Mayne J, et al. PCSK9-deficient mice exhibit impaired glucose tolerance and pancreatic islet abnormalities. FEBS Lett. 2010;584(4):701-706.

17. Horton JD, Cohen JC, Hobbs HH. Molecular biology of PCSK9: its role in LDL metabolism. Trends Biochem Sci. 2007;32(2):71-77.

18. Page MM, Stefanutti C, Sniderman A, et al. Recent advances in the understanding and care of familial hypercholesterolaemia: significance of the biology and therapeutic regulation of proprotein convertase subtilisin/kexin type 9. Clin Sci (Lond). 2015;129(1):63-79.

19. Zaid A, Roubtsova A, Essalmani R, et al. Proprotein convertase subtilisin/kexin type 9 (PCSK9): hepatocyte-specific low-density lipoprotein receptor degradation and critical role in mouse liver regeneration. Hepatology. 2008;48(2):646-654.

20. Ranheim T, Mattingsdal M, Lindvall JM, et al. Genome-wide expression analysis of cells expressing gain of function mutant D374Y-PCSK9. J Cell Physiol. 2008;217(2):459-467.

21. Piao MX, Bai JW, Zhang PF, Zhang YZ. PCSK9 regulates apoptosis in human neuroglioma u251 cells via mitochondrial signaling pathways. Int J Clin Exp Pathol. 2015;8(3):2787-2794.

22. Thompson CB. Apoptosis in the pathogenesis and treatment of disease. Science. 1995;267(5203):1456-1462.

23. Mohamad N, Gutiérrez A, Núñez M, et al. Mitochondrial apoptotic pathways. Biocell. 2005;29(2):149-161.

24. Cotter TG. Apoptosis and cancer: the genesis of a research field. Nat Rev Cancer. 2009;9(7):501-507.

25. Adams JM, Cory S. The Bcl-2 apoptotic switch in cancer development and therapy. Oncogene. 2007;26(9):1324-1337.

26. Pettersson F, Dalgleish AG, Bissonnette RP, Colston KW. Retinoids cause apoptosis in pancreatic cancer cells via activation of RARgamma and altered expression of Bcl-2/Bax. Br J Cancer. 2002;87(5): $555-561$.
OncoTargets and Therapy

\section{Publish your work in this journal}

OncoTargets and Therapy is an international, peer-reviewed, open access journal focusing on the pathological basis of all cancers, potential targets for therapy and treatment protocols employed to improve the management of cancer patients. The journal also focuses on the impact of management programs and new therapeutic agents and protocols on

\section{Dovepress}

patient perspectives such as quality of life, adherence and satisfaction The manuscript management system is completely online and includes a very quick and fair peer-review system, which is all easy to use. Visit http://www.dovepress.com/testimonials.php to read real quotes from published authors. 